\title{
The ROSwell Device
}

\author{
Matt Monfeli, Jerry Miao, Dr. Richard Barker, Dr. Simon Gilroy \\ Botany Department, University of Wisconsin-Madison \\ Madison, WI, United States
}

\begin{abstract}
Plants grown in orbit seem to experience oxidative stress. The gene expression response of the plants to this oxidative stress bears striking resemblance to their response towards dilute hydrogen peroxide $\left(\mathrm{H}_{2} \mathrm{O}_{2}\right)$. Thus, one route towards developing plants better suited to the stresses of spaceflight begins with developing a way to reliably and consistently treat plants with hydrogen peroxide in a laboratory setting. The ROSwell Device embraces this challenge, using 3D-printed components, Arduino and Raspberry Pi microcontroller units, and a solenoid valve mounted on a frame of $80 / 20$ aluminum. The final goal for this device is to construct a reliable method for testing and development of plants designed to grow in space, before sending them into orbit.
\end{abstract}

\section{Background}

Throughout experiments conducted by the Gilroy Lab, Arabidopsis thaliana plants grown onboard the ISS have been shown to experience a novel form of oxidative stress. Through genome ontology enrichment analysis, Dr. Simon Gilroy and Dr. Richard Barker were able to show that this oxidative stress, characterized by an increased production of Reactive Oxygen Species (ROS), generates a very similar genetic response in Arabidopsis as treating the plants with doses of hydrogen peroxide diluted to a concentration of approximately 1 milliMolar. The majority of this data, which focused on 11 core spaceflight response genes, was gathered through the TOAST experiments conducted by the Gilroy Lab (Data is available as GLDS37 at the NASA GeneLab data repository).

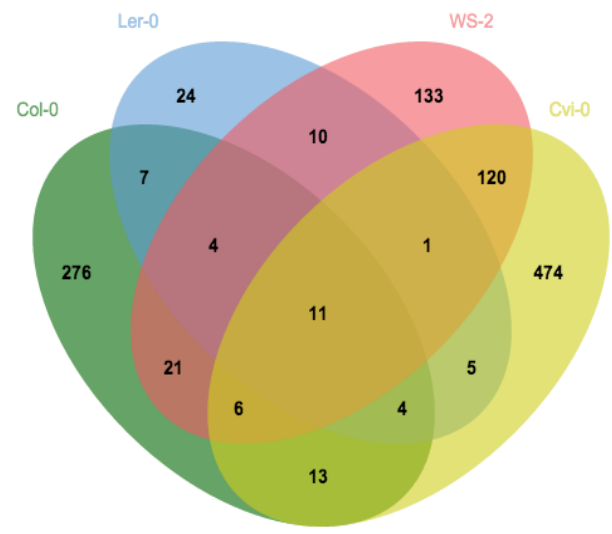

Figure 1: A Venn diagram of all four genotypes, identifying the 11 core spaceflight genes that respond in all variants. Although Cvi-0 has a far larger response than Ler-0, the same 11 genes respond in both when subjected to spaceflight. 


\section{Methods}

In order to create a device that can properly deliver doses of dilute hydrogen peroxide without drowning the Arabidopsis plants and to develop such equipment on a relatively small budget with the goal of making it readily available to the larger scientific community, much of the design of the device was built with open-source materials. Thus, units such as Arduino boards and Raspberry Pi microcontrollers were chosen as well as more accessible fabrication methods such as $3 \mathrm{D}$ printing.

2.1 Frame and mount In order to both protect the device and ensure that it could be mounted at the required angle to facilitate liquid flow, a frame was constructed out of $25 \mathrm{~mm}$ thick 80/20 aluminum. Inside of this frame, the device itself was mounted in a $24 \times 11.5 \times 0.5$ inch piece of acrylic. Using the Universal Laser Systems laser cutter (Figure 2.A), holes were made for both the Petri dish module that would eventually house the plants and the controller screen using the cutter's controller/design software (Figure 2.B). Cuts required 8-10 passes of the laser at maximum power and $1 \%$ speed. (Figure 2.B)

A)
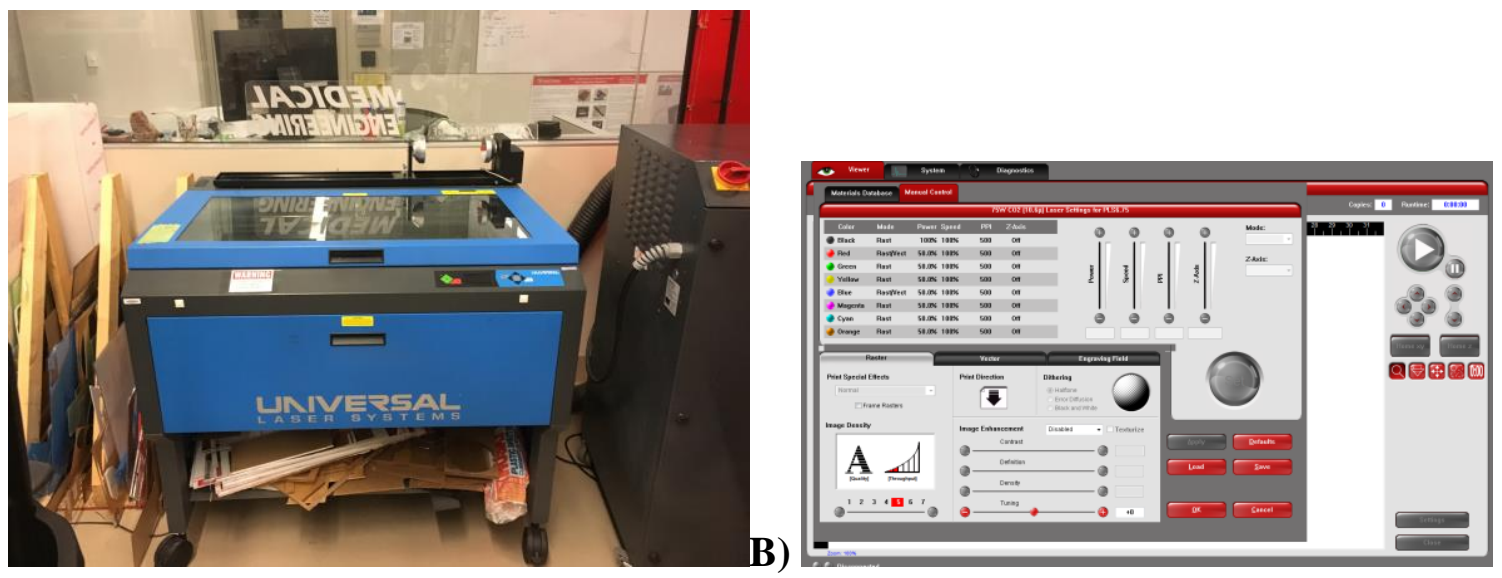

Figure 2

A) The laser cutter. B) Interface for the laser cutter's settings and controls.

2.2 Tubing and control units Although the plant module itself will be discussed in greater detail in the next section, it has serrated hose nozzles on each end which the tubing and valve connect to. These serrated hose nozzles act as both the inlet and the outlet for the fluid. The inlet hose has a $3 / 8$ inch inner diameter and the outlet hose has a $1 / 2$ inch inner diameter. Both hoses are polymer Tygon tubing which can be easily autoclaved for sterilization. The inlet hose attaches to a solenoid valve, located above the plant module. The storage tank for the hydrogen peroxide is in turn located above the solenoid valve. As a result, once the valve is opened the entire system, pictured in Figure 3.A., can be run without a pump, merely using gravity to deliver the hydrogen peroxide. Thus, the primary power draws for the device as a whole are the growth LEDs, the screen, and the control units. Due to the difference in voltage requirements between 
the control units and the solenoid valve, the system demands a power switch, pictured below in Figure 3.B. With the exception of the LEDs and the solenoid valve, all electronics are mounted underneath the acrylic sheet.
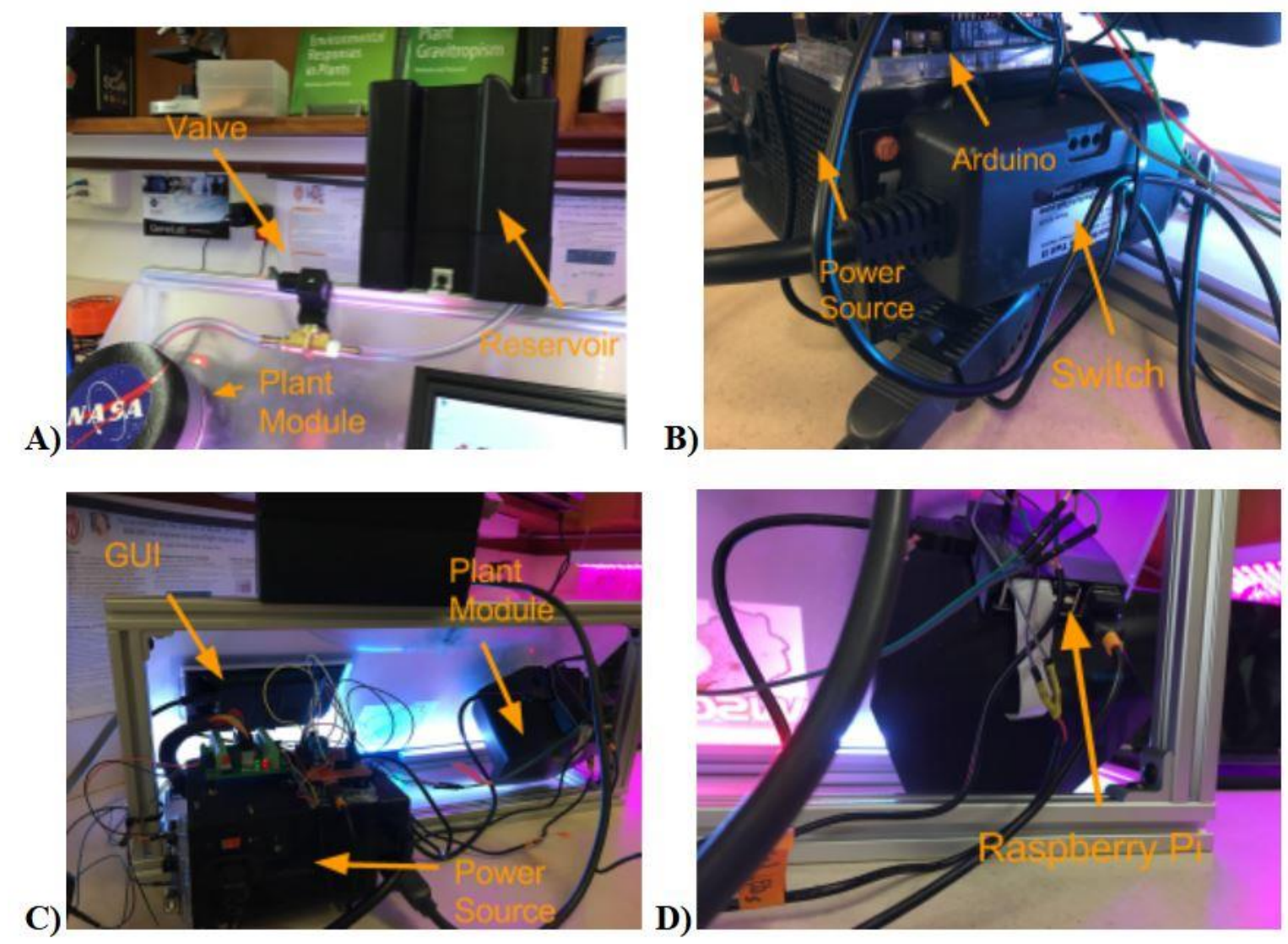

\section{Figure 3}

A) The fluid pathway, located on top of the device. B) The switch needed for the Arduino to send commands to the solenoid valve. C) The system when viewed from the back, showing the wiring connecting all the electronics together. D) The backside of the plant module. The Raspberry Pi is stored on top of it, with the petri dish located on the other side of the acrylic.

2.3 Petri Dish Module The full device within which the Arabidopsis samples are treated and monitored consists of two basic components: The FlashLapse Module and the ROSwell Module. Both were designed in SolidWorks and fabricated using 3D printers.

\subsubsection{FlashLapse module. The FlashLapse module consists of three 3D printed} components, each of which are made in a Makerbot Replicator PLA (Polylactic Acid) extrusion printer. The top component is a simple cap which fits atop the ROSwell module to conceal the plants from outside light. The bottom component serves as a shield from outside light for the plants, a mount for a Raspberry Pi camera, a mount for programmable LED lights, and as a 
mount for the side component. This side component houses the Raspberry Pi controller unit which, when paired with an Arduino, controls both the lighting system and the camera. Thus, what light the plants receive is entirely under the control of the user during experiments. The Raspberry Pi camera can be used to conduct time-lapse photography of the Arabidopsis. When botanists analyze these images with measurement software, growth of the test subjects can be tracked over time.
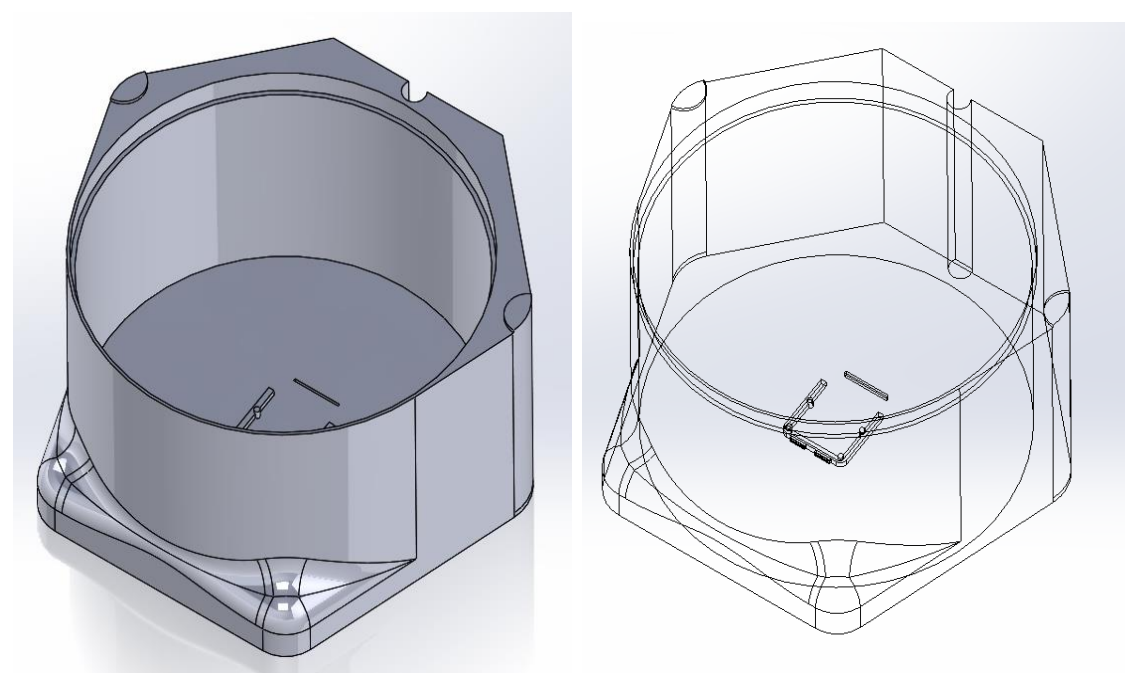

\section{Figure 4}

The FlashLapse bottom component. The Raspberry Pi camera is mounted on the square edges in the center, with the housing for the Pi itself sliding into the circular groove on the far side. The design was altered slightly for the ROSwell Device such that the pieces fit together with the acrylic sheet in the middle.

2.3.2 ROSwell module. The ROSwell module serves as a mount for the petri dish tray and lid, as a connection between the FlashLapse base and lid, and as both an inlet and outlet for the hydrogen peroxide. The petri dish will contain the plant samples, which will be rooted in an agar growth media. The FlashLapse unit was designed as part of a separate project and then adapted to fit this project. 
A)
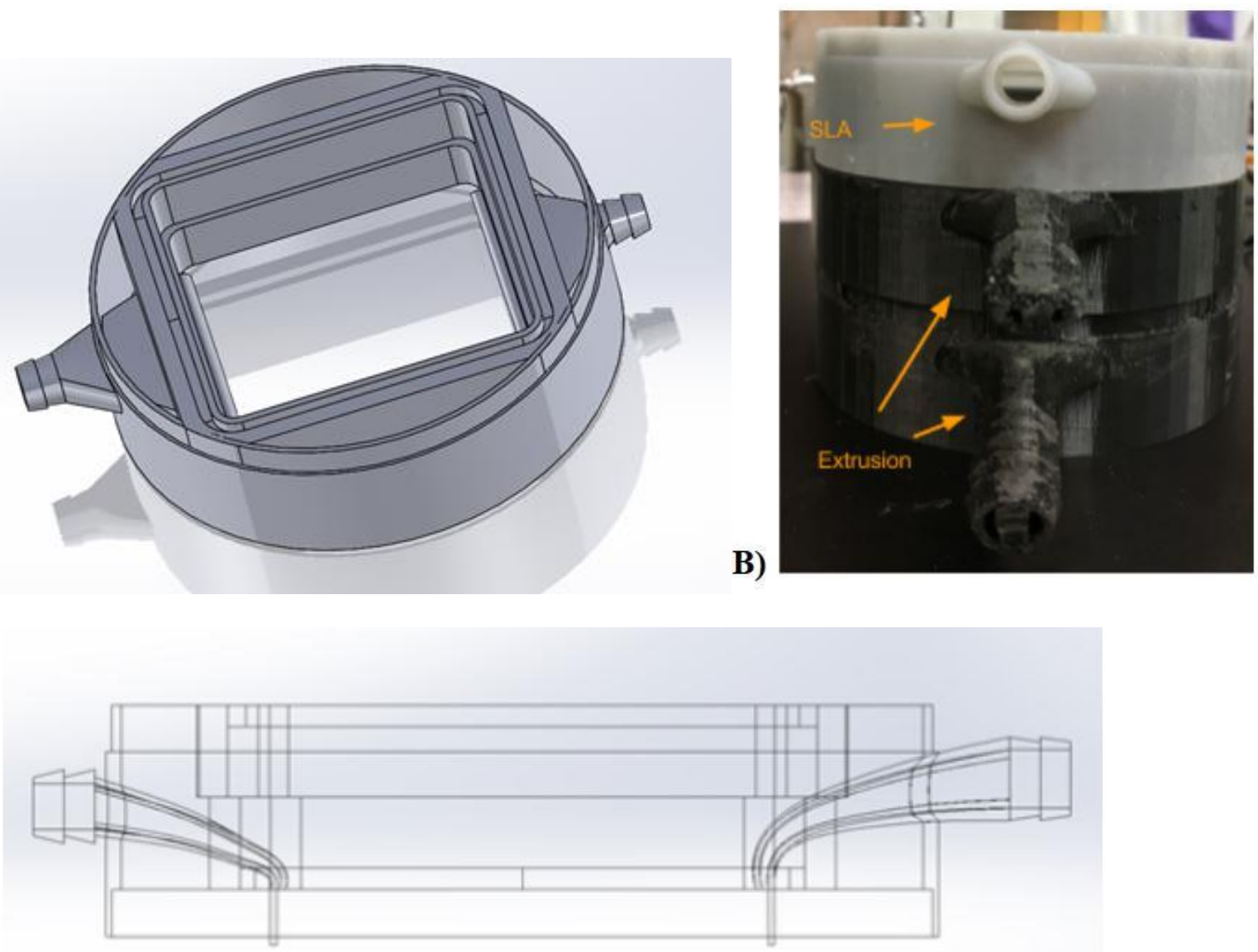

C)

\section{Figure 5}

A) The fully assembled CAD file of the ROSwell module. B) Attempts at printing the ROSwell module with an extrusion printer compared to a stereolithography printer. The hose nozzles on the extruded parts not only collapsed but also had supports blocking the channel. C) The fluid channels cut through the middle of the frame which holds the petri dish. On the top and bottom are edges within which the petri dish lid and tray fit, respectively.

While the module is an assembly of many parts in the SolidWorks design, it is actually fabricated as a single unit. Initial attempts were made to construct the device using a simple PLA extrusion printer; however, the dimensions of the fluid channels required a more exact means of production. Thus, the final version was built using a FormLabs Form2 Stereolithography (SLA) printer, which required no supports inside of the fluid channels during printing. Both ends of the ROSwell module have serrated hose nozzles for attaching tubing. From there, both the inlet and outlet channels cut through the middle of the device, as can be seen in Figure 5.C. These channels gradually loft into a rectangular exit, turning a quarter revolution such that they face the agar when the fluid exits them. In order for the treatment of the plants to be consistent across the petri dish, there must be enough pressure on the fluid as it enters the chamber such that a uniform 
sheet is created. In order to generate this small but necessary pressure, there must be a slight drop in area between the circular entrance of the fluid inlet and its rectangular exit. To keep the fluid sheet in contact with the agar, a small protrusion was added which digs down into its surface. The combined lofted inlet and agar edge can be seen below in Figure 6.A. Due to the width of a petri dish, and the diameter of the tubing, the exit of the fluid inlet is restricted to just $0.5 \mathrm{~mm}$ wide. The precision needed for this dimension restriction requires an SLA printer, rather than a simple extrusion printer.
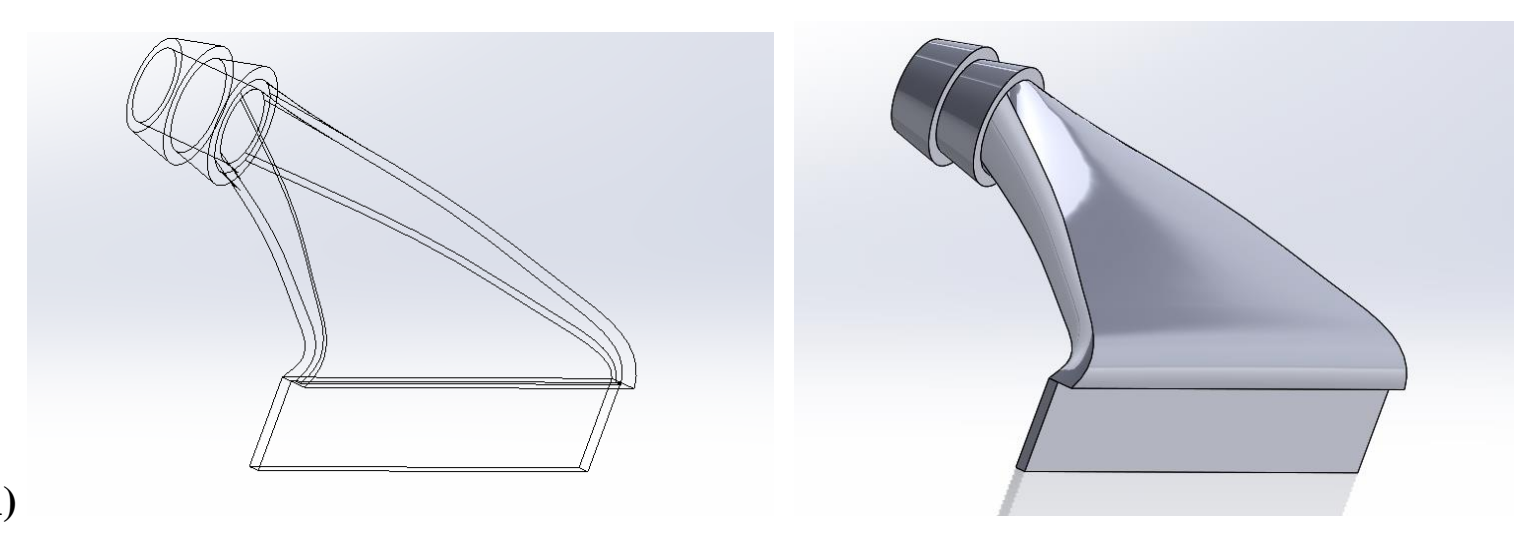

A)

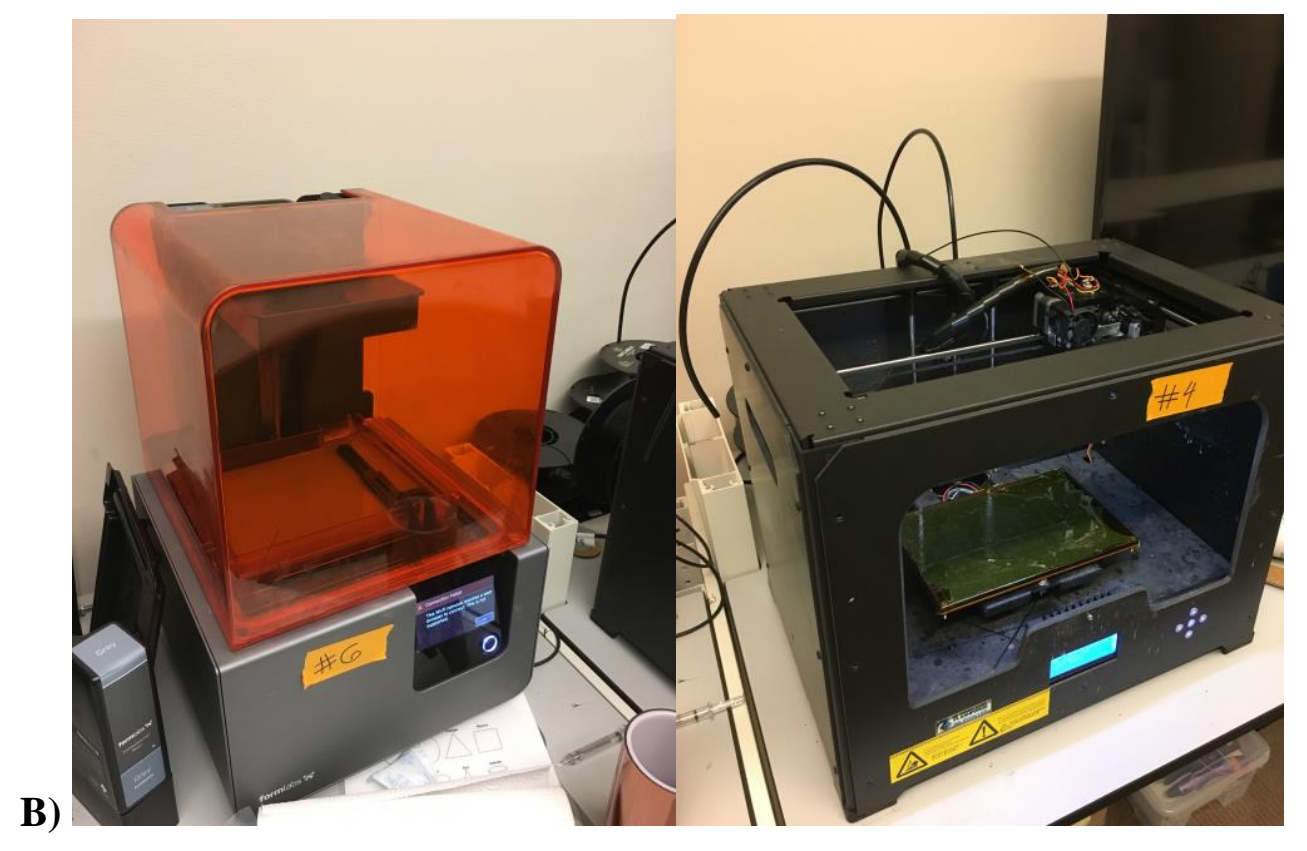

\section{Figure 6}

A) The CAD file for the fluid inlet. Although the dimensions are slightly greater, the outlet has the same basic design as well. The image on the left shows that the inside is indeed hollow, containing the fluid channel. B) From left: FormLabs Form 2 SLA Printer, MakerBot Replicator. 


\subsection{Display screen and graphical user interface}

In order to make the ROSwell device more user-friendly, a small screen was added next to the device, with a graphical user interface (GUI), borrowed from the ongoing FlashLapse project. Following a similar protocol as the petri dish module, a cut was made in the acrylic using the same laser cutter, before placing the device inside this cut and securing it to the surrounding edges, mostly using hot glue.

A)

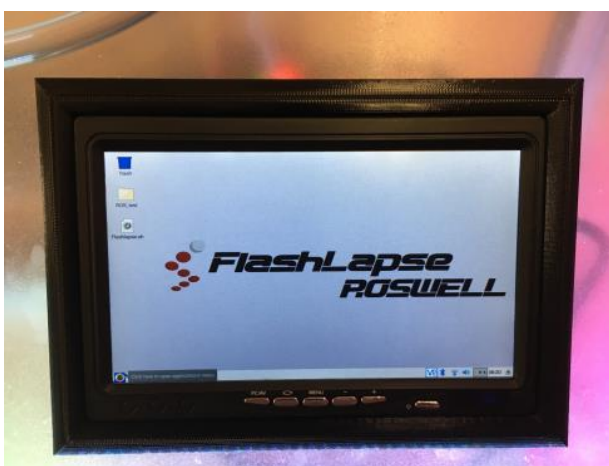

B)

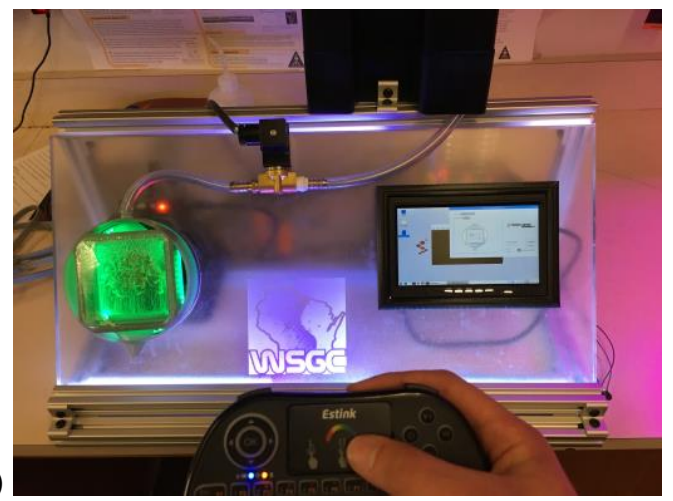

\section{Figure 7}

A) The graphical user interface. B) Using the GUI with a remote control, lights can be adjusted. In this image, the lights are set to green and the petri dish module's cap has been removed. The lights can also be set to red, blue, white, and infrared. The Raspberry Pi camera has an infrared lens as well.

\section{Functionality}

Mechanically, the design functions as planned. All components function to expectations. With programming, the lights can be set to a frequency of the user's choosing more specifically than through the GUI, and images can be easily recorded. As the solenoid valve is controlled by the Arduino, it too is programmable. Thus, the entire fluid pathway of the system functions as intended. The hydrogen peroxide is dispersed evenly across the agar of the petri dish such that a thin sheet of fluid is formed, contacting all plants evenly. The fluid then exits through the outlet channel, ensuring that the plants are not drowned. All components that are along the fluid pathway can be sterilized without causing damage. As previously mentioned, the tubing is simply put into an autoclave. However, the 3D printed parts cannot survive this, and long-term exposure to ethanol proved to be somewhat corrosive for parts from the SLA printer. In testing, bleach left crystals large enough to block the fluid channels once it dried. However, exposure tests conducted over the course of several weeks showed that chlorine gas seemed to have no adverse effects on the material, and thus will be used as the primary sterilization method before experiments. The parts also showed no negative responses to the $1 \mathrm{mM}$ hydrogen peroxide they will be exposed to during testing. 


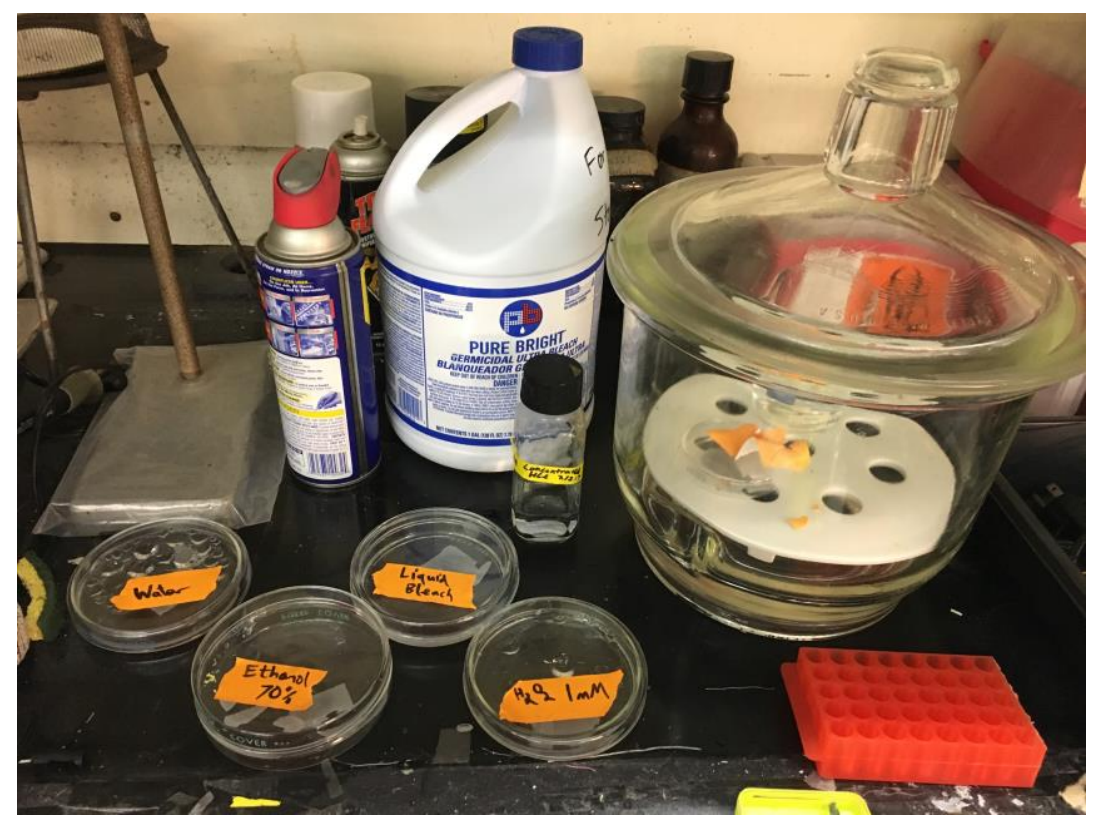

\section{Figure 8}

Exposure tests of the SLA printer's resin to various methods of sterilization and chemicals it will be exposed to in experiments. From left to right (labelled): water (control), ethanol at $70 \%$ concentration, liquid bleach at $50 \%$ concentration, hydrogen peroxide at a concentration of $1 \mathrm{mM}$ (what the plants will be treated with), and an exposure to chlorine gas in the glass container.

Unfortunately, it has proven difficult to keep the plants from washing away with the fluid during testing. With Arabidopsis samples that have grown more than several weeks, the roots are strong enough that this is never a problem. However, the ultimate goal is to perform testing on a sample that is younger, and so this issue cannot be ignored. Mounting the device at an angle instead of vertically has somewhat fixed the problem, though not entirely. The only other noticeable problem for the system currently is the cost of printing the ROSwell module. Each part costs roughly $\$ 30$, and SLA printers are not as common as extrusion printers outside of well-funded laboratories, cutting down on the open-source ability of this device. Thus, some time will likely be dedicated to trying to design a module that can be made through extrusion in the near future. Given the dimensional constraints of the fluid inlet, whether or not this will be successful remains to be seen.

\section{Future goals for project}

While the next prototype is already being designed to exert less force on the plants, the most immediate goal for the project going forward is to begin running multi-day and perhaps multiweek experiments. Slowing this process is the learning of Arduino programming that is currently taking place. With this knowledge, image capture and valve function can occur in unison, allowing the device to function without constant user input. After an initial round of tests are 
completed, the next task is to scale up the design, from a single plant module to twelve. Development for this larger unit is already well underway, and construction of its considerably larger frame will begin in a few weeks. Assuming that meaningful data can indeed be gained from this device, the end goal is to use it on NASA target crops, such that a better model of the stresses of spaceflight can be applied to plants here on Earth other than Arabidopsis.

\section{Acknowledgements}

Dr. Richard Barker- Recognized the oxidative stresses that plants experience in space and made me aware of how to replicate them on Earth. He also acted as an invaluable teacher and mentor, gave assistance with the botany side of this experiment, as well as access to the many resources of the Gilroy Lab and the Wisconsin Institute for Discovery.

Dr. Simon Gilroy- For acting as a mentor and opening up resources of the Gilroy Lab.

Travis Tangen- Provided access to 3D printers and laser cutters. Supplied much of the materials that otherwise would have been bought separately, and many other resources related to the engineering side of this project.

Rob Swader- Provided access to CAD software and mentoring for the engineering components of this project.

Ben Cox- Provided mentoring for use of CAD software, which was invaluable as I had never used any before this project.

Jerry Miao- Developed the FlashLapse unit and helped tremendously with wiring the various units together. He has also been extremely helpful in my learning how to write code and solder circuits.

Jeff Vogtschaller- Provided assistance in assembling the aluminum frame for this project.

Beau Gotthardt- Provided assistance in learning how to use an SLA printer.

\section{Materials/Resources Required for Project}

- Makerbot Replicator PLA/ABS extrusion 3D Printer

- PLA filament, any color other than transparent $(1 \mathrm{~kg})$

- FormLabs Form2 SLA 3D Printer

- FormLabs Standard Resin, black or grey (1L)

- Tygon $1 / 2$ " ID Polymer Tubing (3ft)

- Tygon $3 / 8$ " ID Polymer Tubing (3ft)

- Universal Laser Systems Laser Engraver/Cutter

- Raspberry Pi 3

- Arduino Uno (2)

- Adafruit Neopixel Programmable LED Strip (3ft+) 
- Solenoid Valve

- Serrated hose nozzle adapter for $1 / 4$ " pipe to $3 / 8$ " tubing (2)

- 4.5 x 7 inch Screen

- Computer with SolidWorks, Makerbot, PreForm software downloaded

- 25 x $25 \mathrm{~mm} \mathrm{80/20} \mathrm{aluminum} \mathrm{(15ft)}$

- Circular saw for cutting 80/20 aluminum

- Belt sander or Deburring tool for smoothing cuts on 80/20 aluminum

- 80/20 Corner Brackets, Bolts, Washers

- Adafruit Industries Jumper Wire

- Electronic/Salon 24/20-pin ATX DC Power Supply Breakout Board

- Power Source

- PowerSwitch Tail II

- Rinse bath for SLA prints

- 24 x 11.5 x 0.5 inch Acrylic Sheet 